\title{
Postabortion Care: Going to Scale
}

\section{By Saumya \\ RamaRao, \\ John W. Townsend, \\ Nafissatou Diop \\ and Sarah Raifman}

Saumya RamaRao is senior associate, and John W. Townsend is vice president, both at the Population Council, New York. Nafissatou Diop is coordinator, United Nations Population Fund-United Nations Children's Fund Joint Programme on Female Genital Mutilation/ Cutting, New York. Sarah Raifman is staff assistant, Population Council, New York.
Millennium Development Goal 5 aims to reduce the 1990 level of maternal mortality by $75 \%$ by 2015 . The major direct causes of maternal mortality are well documented: Most women die from severe bleeding, infections, eclampsia, obstructed labor or the consequences of unsafe abortion. ${ }^{1}$ Evidence-based interventions exist to address each of these causes. Safe abortion where legal, family planning and postabortion care-the management of the patient once she presents to a health provider with complications caused by either a miscarriage or an induced abortion-are interventions that provide options for women to ensure that every child is a wanted child. Given that 13\% of maternal deaths result from unsafe abortions, ${ }^{2}$ postabortion care has the potential to prevent maternal deaths. Postabortion care is especially relevant in countries where abortion is restricted, but is useful even in countries where abortion is legal but access to safe services is limited.

For the past two decades, efforts have been made to design service delivery models that incorporate the specific elements that postabortion care programs should provide. ${ }^{3,4}$ Currently, there are two main models-one developed by the PAC Consortium and one developed by the United States Agency for International Development (USAID); there are significant overlaps between the two. The model advocated by the PAC Consortium includes five elements: partnerships between communities and service providers; client-centered counseling at appropriate times during service delivery; treatment with emphasis on pain management; family planning; and links to reproductive and other health services. ${ }^{3}$ The USAID model has three components: emergency treatment; family planning and STI and HIV services; and community empowerment via community awareness and mobilization. ${ }^{4}$ Countries have been able to draw upon the two models when designing and implementing postabortion care services. Created to facilitate scale-up and increase access to postabortion care, the models provide guidance on how services could be designed and delivered; how postabortion care could be integrated with other health services, including family planning; and how to develop the partnerships required with the communities that are the ultimate beneficiaries of service improvements.

The ultimate goal for postabortion care programs is scaling up-increasing service coverage by replicating the pilot program or moving a given service from its pilot orientation and integrating it into the existing health system. In order for a service model to go to scale, it must demonstrate feasibility, efficacy and potential for sustainability, in addition to being client-oriented. Furthermore, the new service must be written into policy documents; service guidelines and standards must be developed and deployed; providers must be trained; and facilities and staff must be reoriented in providing this new service. Scaling up is vitally important because it improves access for women in need of postabortion care, which ultimately contributes to declines in maternal mortality.

Operations research has generated evidence on a variety of specific service delivery questions to help policymakers and program managers design and deliver postabortion care services. These investments in research will pay off if health programs are able to use the findings to improve service delivery, resulting in better health and well-being for sexually active women who have resorted to abortion because of a mistimed or unwanted pregnancy or who have miscarried a wanted pregnancy.

In this comment, we discuss the complementary efforts required to ensure that research findings are utilized and postabortion care services are scaled up. Complementary efforts range from identifying champions and advocates for postabortion care to providing technical assistance for replication and scale-up. Our conclusions draw on experiences in diverse program settings in Sub-Saharan Africa, Latin America and Asia where postabortion care services have been, or have the potential to be, scaled up.

\section{Key Findings from Research}

- The safety of manual vacuum aspiration. Studies have overwhelmingly shown that vacuum aspiration is a safe and effective alternative to dilation and curettage ( $D \& C$ ): A Cochrane Review reported that vacuum aspirations are safe, easy to perform and cause less pain than D\&C. ${ }^{5}$ Furthermore, in resource-constrained settings where a regular supply of electricity may not be available, manual vacuum aspirations are a feasible approach with safe and effective results ${ }^{6,7}$ In addition to being safe and effective, manual vacuum aspiration is more cost-effective than its alternatives because fewer staff are needed, no operating room facilities are required and women need less recovery time at the facility. ${ }^{8}$ Demonstrated increases in quality and safety, combined with lower costs and reduced use of resources, make manual vacuum aspiration an important tool in facilitating scale-up.

- Use of midlevel providers. In some settings, the use of midlevel health practitioners instead of physicians to provide safe manual vacuum aspiration services has helped make uterine evacuations more accessible and available to 
women. Provision of manual vacuum aspiration by midlevel providers has made it possible for postabortion care services to be offered at primary health care facilities that are closer to the communities they serve than are the less common and more distant tertiary facilities, such as hospitals and referral centers, that have doctors on staff. That postabortion care services can be feasibly and safely provided at lower-level facilities by midlevel providers has been amply demonstrated in such countries as Ghana, Kenya, Nepal, Senegal and Tanzania..$^{9-14}$ On the basis of this evidence, other countries are moving to decentralize services. ${ }^{15}$ With increasingly decentralized service provision and success in using midlevel providers for postabortion care, the possibility of scaled-up services becomes even more feasible.

- Counseling and pain management. The elaboration of the two models was instrumental in focusing attention on two critical components of postabortion care-counseling and pain management. Furthermore, the introduction of manual vacuum aspiration included training that emphasized appropriate pain management and counseling. Specific guidance was developed on appropriate types of medications, their dosage and when they can be administered. ${ }^{16}$ When manual vacuum aspiration is introduced as a package with pain management and counseling, research indicates that the pain experienced by clients decreases. ${ }^{17}$ However, the evidence suggests that pain management may not be a well-established practice for a variety of reasons, including short supplies of necessary medications and resources, negative provider attitudes toward women who have had an abortion or poor adherence to clinical protocol. ${ }^{18-22}$

Counseling is an essential feature of the care-giving process that complements and contributes to pain management. This exchange of information between client and provider (and increasingly, other family members) covers many topics, such as procedures to be followed before, during and after evacuation and contraceptive options. Empathetic reassurances by the provider during counseling can also have therapeutic effects on pain. ${ }^{23}$ When postabortion care goes to scale, the inclusion of counseling and pain management in the service model ensures that these aspects of service quality, a critical part of the client's right to empathetic and humane care, are not lost during integration into the health system.

- Infection prevention. When manual vacuum aspiration was introduced, training programs on the procedure systematically integrated infection prevention into their curricula, including procedures for sterilizing and processing equipment; disposal; and such standard infection prevention measures as the use of sterile gloves and drapes. This emphasis has been useful in improving provider knowledge and adherence to infection procedures. ${ }^{14}$ When programs go to scale, clear guidance on infection prevention is critical to ensure that services are provided as safely and as well as they were during the pilot phase.

- Integrating family planning services into postabortion care.
Over the years, theoretical and programmatic rationales have been put forth to ensure that postabortion clients receive contraceptive information and services if they desire. ${ }^{3,24}$ Ample evidence exists from many countries that integrating family planning services into postabortion care is feasible and can be carried out in various ways. ${ }^{25-28}$ As countries issue policy guidelines on integrated service delivery, such results as these are useful in informing implementation plans from the tertiary to primary health care levels.

- Cost of postabortion care services. The finding that postabortion care services can be provided within existing health system budgets has been persuasive for program planners and policymakers. The methodology for estimating the costs of providing postabortion care services has been developed and refined, generating estimates of cost per patient, as well as cost to the facility and the health system. ${ }^{29-31}$ Research has documented that costs can be reduced by switching to vacuum aspiration for uterine evacuation (because it limits the length of hospital stays and is less expensive than D\&C) and by having midlevel providers rather than clinicians provide services. ${ }^{30,32}$ For example, an economic analysis conducted in the late 1990s in Oaxaca, Mexico, estimated that the total cost per patient was reduced from US\$265 to US\$18 after the introduction of manual vacuum aspiration and the associated quality improvement package. ${ }^{32}$ With a greater accumulation of experience in providing postabortion care services across many settings, it is clear that postabortion care services can be incorporated into existing health systems without too much additional outlay. These cost benefits are crucial to providing the evidence for sustainability needed to plan for the scale-up of postabortion care services.

\section{Remaining Challenges}

Despite these many gains and successes, there continue to be areas that require additional strengthening. As postabortion care services transition from pilot programs to scaled-up services, some areas will require further technical support and attention for the integration of these services into the health system to be successful.

The most significant challenge is ensuring that every woman who receives postabortion care also receives information on her return to fertility and contraceptive options, and contraceptive services if she so desires. Although there have been many documented models of how family planning services can be integrated with postabortion care services, implementation remains weak. At the health system level, implementation is hindered by poor contraceptive logistics and lack of clear national policies and guidelines on integrated services; at the facility level, disruptions in contraceptive supplies and separate locations for family planning and postabortion care services are common problems; and at the provider level, constraints include poor skills in counseling clients and lack of supervisory support to overcome service challenges. ${ }^{24,27}$ There is empirical evidence that when contraceptives are available at the site of postabortion care ser- 
vices and women are counseled before discharge, they are more likely to accept a method than they are when those services are not provided. ${ }^{33}$

A second significant challenge is providing a consistent and reliable supply of equipment and associated medications for manual vacuum aspiration, without which scaleup is impossible. This has been a particular problem in francophone Africa, where many countries lack a functioning supply system. ${ }^{25}$ Typically, equipment for manual vacuum aspiration is distributed by development partners during the training of providers when postabortion care services are first introduced. Rarely do ministries of health purchase the equipment for the providers undergoing the training. Even in countries where postabortion care services have been provided as part of the national program for a few years, such as in Burkina Faso, Guinea and Senegal, health facilities may have difficulties in reordering equipment for manual vacuum aspiration if this equipment is not listed on the essential drugs list or in central procurement and distribution guidelines. Temporary solutions, such as obtaining supplies from private sources, have been adopted in some countries, but these types of measures indicate challenges to ensuring sustainability. In addition, consistent availability of pain medications and multiple kinds of contraception for postabortion family planning services is vital. National systems that have incorporated supplies for manual vacuum aspiration into the essential drug list and have more streamlined procedures have an increased likelihood of success in scale-up. Reproductive health advocates have argued for the inclusion of reproductive health drugs and supplies, including equipment for manual vacuum aspiration, into countries' essential drugs list. ${ }^{34}$

A third challenge is ensuring the quality of postabortion care services once they have been introduced. Special training strategies are required for providers and their supervisors so that emergency services, counseling and family planning services continue to be emphasized after training. Instituting initial supervision for providers-in-training, as well as standard training for trainers, is essential to the overall quality and sustainability of a large-scale postabortion care service program. In Bolivia, for example, supervisors needed to be trained to be trainers and to ensure that providers maintained their skills. ${ }^{35}$ Ongoing supervision, refresher training, program monitoring and periodic evaluations are useful mechanisms for assuring quality. Furthermore, incorporating theoretical and practical training on postabortion care in preservice curricula in medical and nursing schools will foster momentum for scaling up services. The earlier that techniques such as manual vacuum aspiration and electric vacuum aspiration are incorporated into existing curricula, the better prepared new health providers will be to provide postabortion care when they enter the service; in-service training would then take the form of refresher training. In a similar vein, the more quickly new methods of uterine evacuation, such as use of misoprostol, can be incorporated into service regimens and preservice curricula, the greater the repertoire of available options will be for women and providers.

\section{What's Required for Scale-Up}

Several postabortion care service programs have gone to scale, most of them in Africa. For example, in Senegal, the ministry of health worked with Management Sciences for Health to extend postabortion care services to urban and rural facilities in 23 health districts in a series of phases and interventions. ${ }^{36}$ Intrahealth's PRIME II Project contributed to significant progress in expanding the availability of high-quality postabortion care and family planning services at the primary care level in Kenya. ${ }^{37}$ Development partners continue to provide technical support in a number of countries scaling up postabortion care services. The following are vital to the successful scaling up of postabortion care projects:

- Existence of a policy champion. The presence of a policy champion is essential for advocacy, setting the stage for the introduction of postabortion care services and subsequent scaling-up. The policy champion plays an important role in communicating with a variety of audiences, building a coalition of advocates, policymakers, program managers and service providers to establish a welcoming environment for introduction of an intervention, pilot testing it and taking it to scale. ${ }^{38}$ The provision of postabortion care services and the potential for scale-up are highly dependent on existing governmental policies and the environment within which policies are formed and revised. The policy champion uses the available evidence to persuade stakeholders representing different constituencies (e.g., national policy, subnational program, community advocates) to take concerted action. Along with the champion, development agencies support sensitization and awarenessraising activities, as well as facilitate dialogue between stakeholders and key decision makers. Policy champions are most effective when they are able to work in an environment that is receptive or neutral to the proposed policy or service change. Technical assistance and support agencies play an important role in providing policy champions with relevant support and information.

- Empirical evidence of need for postabortion care and program response. Statistics from health facilities on women seeking services for abortion complications can be used to help policymakers understand the nature and volume of demand for postabortion care. Information on how a program provides care to women in need gives policymakers a description of current service practices, including gaps. However, empirical findings need to be translated into actions that policymakers and program managers can take to improve services.

For example, global research across regions has demonstrated a considerable demand for postabortion care services. The World Health Organization estimates that $10-50 \%$ of the 20 million unsafe abortions that occur annually worldwide require medical care; some women who experience spontaneous abortion also need treatment. ${ }^{39}$ 
In Ethiopia, researchers reported that almost 58,000 women sought care for complications of induced or spontaneous abortion in 2008; the case-fatality rate among women seeking postabortion care in public hospitals was 628 per 100,000 , leading the authors to conclude that further expansion and strengthening of postabortion care and safe abortion services is needed to reduce mortality and morbidity and reduce the financial burden on hospitals. ${ }^{40}$ Furthermore, a growing body of evidence on the feasibility and sustainability of postabortion care procedures has documented appropriate program responses. These types of information, including data on cost and cost-effectiveness, provide decision makers with a sufficient rationale to proceed with scaling-up.

Data from multiple sources provide a comprehensive picture of beneficiary needs; program responses and gaps; and funding requirements that policymakers can use as evidence to support scale-up of services. For example, studies in Egypt, Kenya, Nigeria and Zimbabwe found provision of postabortion care to be effective; ${ }^{41}$ in many settings, women have reported appreciating the greater personal attention they receive when manual vacuum aspiration is provided in an office setting or lower-level facility. ${ }^{6}$

- A phased approach. Several of the first countries to introduce postabortion care went through pilot phases to test the provision of services before broader implementation. A phased approach is a useful for a new service, especially one that is socially and culturally sensitive in many parts of the world. In such countries, key national stakeholders may gather to discuss results from operations research and pilot studies, providing a forum for transparent communication, strategizing programmatic responses, planning the timing of policy changes, building consensus and enlarging the pool of invested stakeholders.

Operations research and the use of pilot studies have helped to guide strategies and actions related to postabortion care services delivered in hospitals and health facilities in many countries. A pilot operations research phase is important for demonstrating feasibility and effectiveness with rigorous documentation, for selecting the most appropriate models for intervention and for facilitating the introduction of services. The results of the operations research phase are critical for formulating plans for the scale-up phase. ${ }^{42}$ For example, after the first operations research study in Senegal demonstrated feasibility of postabortion care provision in 1997-1998, national postabortion care protocols and norms were developed and disseminated and services were eventually decentralized to four regional hospitals and one district health center.

- Partnerships and community mobilization. In successful efforts to scale up postabortion care services, policy champions and other stakeholders seek and identify ideal platforms for launching the policy or program change. Identifying the most appropriate partners and ensuring their active participation is crucial for a successful scale-up.

In some countries, postabortion care is first introduced at the tertiary level; in others, at the primary level. In Mali and Togo, where postabortion care was introduced in 2006, decentralized services were instituted at the beginning of the process. Some countries (such as Burkina Faso, Niger and Senegal) adopted a centralized approach to the scale-up of postabortion care services, with guidance flowing from the national to subnational levels, while in Mali, the decentralized local authorities are responsible for setting their own priorities and finding program funding. The decentralized approach, which empowers local authorities to develop postabortion care services, offers the possibility of quickly moving from dependency on outside funding to easily involving local communities.

Some countries have been able to fold postabortion care into a larger program with a broader focus. In Nepal, national postabortion care services were established within an emergency obstetric care program. The integration of these programs allowed for the successful introduction of nurse-led postabortion care services. The project's success led to the creation of national policy to expand the postabortion care program throughout Nepal. ${ }^{13}$

By engaging with communities-women, men and community leaders-policymakers and program designers can build momentum for scaling up postabortion care services. Newer service delivery models emphasize community mobilization not only to advocate for postabortion care services but also to raise community awareness of the need for such services, to reduce stigma and to find appropriate roles for all engaged community members.

\section{Conclusion}

Despite the advances made in the medical management of postabortion complications and the accumulation of evidence on feasibility and sustainability of program responses, much remains to be done to ensure that the momentum generated does not flag. Needs have been identified, interventions are well-known and know-how is available for program design and implementation. Joint statements from international organizations and leaders, such as the one on the importance of strengthening the family planning component of postabortion care issued in September 2009 by the International Federation of Gynecology and Obstetrics, International Confederation of Midwives, International Council of Nurses and USAID (http://www.usaid.gov/our_work/global_health/pop/ techareas/pac/fp_component.pdf), provide the necessary support and impetus. Continued progress in this important reproductive health area will require sustained advocacy, political will and steady service provision.

\section{REFERENCES}

1. Ronsmans $C$ et al., Maternal mortality: who, when, where and why, Lancet, 2006, 368(9542):1189-1200.

2. Singh S et al., Abortion Worldwide: A Decade of Uneven Progress, New York: Guttmacher Institute, 2009.

3. Corbett MR and Turner KL, Essential elements of postabortion care: origins, evolution and future directions, International Family Planning Perspectives, 2003, 29(3):106-111.

4. Solo J et al., USAID Postabortion Care Strategy Paper, Washington, DC: 
United States Agency for International Development (USAID), 2004.

5. Forna F and Gülmezoglu AM, Surgical procedures to evacuate incomplete abortion, Cochrane Database of Systematic Reviews, 2001, Issue 1, No. CD001993.

6. Baird TL and Flinn SK, Manual Vacuum Aspiration: Expanding Women's Access to Safe Abortion Services, Chapel Hill, NC, USA: Ipas, 2001.

7. Cisse CT, Faye KG and Moreau JC, First-trimester abortion at University Hospital Center in Dakar, Senegal: utility of manual vacuum aspiration, Médicine Tropicale, 2007, 67(2):163-166 (in French).

8. Bradley J et al., A comparison of the costs of manual vacuum aspiration (MVA) and evacuation and curettage ( $E$ and $C$ ) in the treatmen of early incomplete abortions in Kenya, Journal of Obstetrics and Gynaecology of Eastern and Central Africa, 1993, 11(1):12-19.

9. Population Council, Improving the Counseling and Medical Care of Post Abortion Patients in Egypt: Final Report, Cairo: Population Council, 1995 , p. 215

10. Brookman-Amissa E et al., Decentralizing postabortion care in Africa: a call to action, African Journal of Reproductive Health, 1999, 3(1):109-114.

11. Billings D et al., Midwives and comprehensive abortion care in Ghana, in: Huntington D and Piet-Pelon NJ, eds., Postabortion Care: Lessons from Operations Research, New York: Population Council, 1999, pp. 141-158.

12. Extending Service Delivery, Pathfinder International, A description of the private nurse midwives networks (clusters) in Kenya: a best practice model, Best Practices Series Report, 2007, No. 2.

13. Basnet I et al., Evolution of the postabortion care program in Nepal: the contribution of a national safe motherhood project, International Journal of Gynaecology \& Obstetrics, 2004, 86(1):98-108.

14. Wanjiru M et al., Assessing the feasibility, acceptability and cost of introducing postabortion care in health centres and dispensaries in rural Tanzania, Washington, DC: Population Council, 2007.

15. McDougall J et al., Determinants of contraceptive acceptance among Cambodian abortion patients, Studies in Family Planning, 2009, 40(2):123-132

16. Castleman L and Mann C, Manual Vacuum Aspiration (MVA) for Uterine Evacuation: Pain Management, Chapel Hill, NC, USA: Ipas, 2002.

17. Dabash R et al., Taking postabortion care services where they are needed: an operations research project testing PAC expansion in rural Senegal, Washington, DC: Population Council, 2003

18. Langer A et al., Improving postabortion care with limited resources in a public hospital in Oaxaca, Mexico, in: Huntington D and PietPelon NJ, eds., Postabortion Care: Lessons from Operations Research, New York: Population Council, 1999, pp. 80-107.

19. Salter C, Johnson HB and Hengen N, Care for postabortion complications: saving women's lives, Population Reports, 1993, Series L, No. 10.

20. Htay TT, Sauvarin J and Khan S, Integration of post-abortion care: the role of township medical officers and midwives in Myanmar, Reproductive Health Matters, 2003, No. 11, pp. 27-36.

21. Solo J, Easing the pain: pain management in the treatment of incomplete abortion, Reproductive Health Matters, 2000, No. 8, pp. $45-51$.

22. Fetters T, Tesfaye S and Clark KA, An assessment of postabortion care in three regions in Ethiopia, 2000 to 2004, International Journal of Gynaecology \& Obstetrics, 2008, 101(1):100-106.

23. Piet-Pelon, N, Conclusion, in: Huntington D and Piet-Pelon NJ, eds., Postabortion Care: Lessons from Operations Research, New York: Population Council, 1999, pp. 198-212.

24. Curtis C, Huber D and Moss-Knight T, Postabortion family planning: addressing the cycle of repeat unintended pregnancy and abortion, International Perspectives on Sexual and Reproductive Health, 2010, 36(1):44-48

25. Dieng T et al., Assessment of progress of the postabortion care ini- tiative in francophone Africa, Washington, DC: Population Council, 2008 .

26. Solis F et al., Situation analysis of the use of contraception in postpartum, postabortion, and prevention of mother-to-child HIV transmission, Nicaragua, Washington, DC: Population Council, 2008.

27. Rivero-Fuentes ME et al., Haiti: situation analysis of the use of contraception in postpartum, postabortion, and prevention of mother-tochild HIV transmission, Washington, DC: Population Council, 2008.

28. Youssef $\mathrm{H}$ et al., Linking family planning with postabortion services in Egypt: testing the feasibility, acceptability and effectiveness of two models of integration, Washington, DC: Population Council, 2007.

29. Abernathy M et al., A Guide to Assessing Resource Use for the Treatment of Incomplete Abortion, Carrboro, NC, USA: Ipas, 1993.

30. Johnston HB, Gallo MF and Benson J, Reducing the costs to health systems of unsafe abortion: a comparison of four strategies, Journal of Family Planning and Reproductive Health Care, 2007, 33(4):250-257.

31. King T, Benson J and Stein K, Comparing the Cost of Postabortion Care in Africa and Latin America: The DataPAC Project, New York: Population Council, 1997.

32. Brambila C et al., Estimating costs of postabortion services at Dr. Aurelio Valdivieso General Hospital, Oaxaca, Mexico, in: Huntington $\mathrm{D}$ and Piet-Pelon NJ, eds., Postabortion Care: Lessons from Operations Research, New York: Population Council, 1999, pp. 108-124.

33. Abdel-Tawab N, Challenges and opportunities in providing postabortion family planning services, paper presented at the XXVI IUSSP International Population Conference, Marrakech, Morocco, Sept. 27-Oct. 2, 2009

34. Hutchings J, Neroutsos K and Donnelly K, Making the list: the role of essential medicines lists in reproductive health, International Perspectives on Sexual and Reproductive Health, 2010, 36(4):205-208.

35. Diaz J et al., Improving the quality of services and contraceptive acceptance in the postabortion period in three public-sector hospitals in Bolivia, in: Huntington D and Piet-Pelon NJ, eds., Postabortion Care: Lessons from Operations Research, New York: Population Council, 1999 pp. 61-79.

36. Thiam FT, Siri S and Moreira P, Scaling up postabortion care services: results from Senegal, Occasional Papers, Cambridge, MA, USA Management Sciences for Health, 2006, No. 5

37. IntraHealth, Voices from the field: scaling-up postabortion care services, PRIME Voices, 2004, No. 26, <http://www.intrahealth.org/page/ scaling-up-postabortion-care-services>, accessed Feb. 18, 2011.

38. Family Health International, Engaging innovative advocates as public health champions, Research Utilization Brief, 2010, <http://www. fhi.org/NR/rdonlyres/eqdet4k5um4nnujqxgdyxgi44rssbvmczrme 7 di4muqqubjffparoroyb2vp2k2iy4odlqr5fepllh/RUchampions.pdf>, accessed Jan. 31, 2011

39. World Health Organization (WHO), Unsafe Abortion: Global and Regional Estimates of Incidence of Unsafe Abortion and Associated Mortality in 2003, Geneva: WHO, 2007.

40. Gebreselassie H et al., Caring for women with abortion complications in Ethiopia: national estimates and future implications, International Perspectives on Sexual and Reproductive Health, 2010, 36(1):6-15

41. Kizza AP and Rogo KO, Assessment of the manual vacuum aspiration (MVA) equipment in the management of incomplete abortion, East African Medical Journal, 1990, 67(11):812-822.

42. Support for Analysis and Research in Africa (SARA) Project, Issues in Postabortion Care: Scaling-Up Services in Francophone Africa, Washington, DC: SARA Project, 2004

\section{Acknowledgments}

This comment was supported in part by funding from the Hewlett Foundation.

Author contact:sramarao@popcouncil.org 